\title{
Recent ion measurements within the modified DPF-I000U facility
}

\author{
Roch Kwiatkowski, \\ Krzysztof Czaus, \\ Elzbieta Skladnik-Sadowska, \\ Marek J. Sadowski, \\ Dobromil R. Zaloga, \\ Marian Paduch, \\ Ewa Zielinska
}

\begin{abstract}
In this note we describe measurements of ion beams emitted along the z-axis of the DPF-1000U facility operated at $23 \mathrm{kV}, 334 \mathrm{~kJ}$, and with the initial deuterium pressure of 1.6-2 hPa. The DPF-1000U device was recently renewed and equipped with a dynamic gas-puff valve placed inside the inner electrode. The investigated ions were recorded by means of ion pinhole cameras equipped with solid state nuclear track detectors of the PM-355 ${ }^{\circledR}$ (PADC) type. The energy spectra of ions were determined using a Thomson spectrometer placed on the symmetry axis at a distance of $160 \mathrm{~cm}$ from the electrodes outlets. The ion images recorded during discharges performed under different experimental conditions show that the ion beams have a complex structure, usually in the form of a central bunch and an annular stream composed of many micro-beams. Energies of the registered deuterons have been in the range of 30-700 keV, while the fast protons (which originated from the hydrogen remnants) had energies in the range of 300-850 keV.
\end{abstract}

Key words: DPF-1000U facility • ion beams • ion energy spectra • ion pinhole images

R. Kwiatkowski ${ }^{\bowtie}$ K. Czaus, E. Skladnik-Sadowska, D. R. Zaloga

National Centre for Nuclear Research (NCBJ),

7 Andrzeja Soltana Str., 05-400 Otwock/Swierk, Poland, Tel.: +48 22718 0417, Fax: +48 22779 3481,

E-mail: Roch.Kwiatkowski@ncbj.gov.pl

\section{J. Sadowski}

National Centre for Nuclear Research (NCBJ),

7 Andrzeja Soltana Str., 05-400 Otwock/Swierk, Poland and Institute of Plasma Physics and Laser Microfusion (IPPLM),

23 Hery Str., 01-497 Warsaw, Poland

M. Paduch, E. Zielinska

Institute of Plasma Physics and Laser Microfusion (IPPLM),

23 Hery Str., 01-497 Warsaw, Poland

Received: 17 June 2014

Accepted: 2 January 2015

\section{Introduction}

The aim of ion measurements at plasma facilities is to identify the various ion species emitted from such devices and to determine their characteristics, such as the energies and the angular distribution. Studies of the fast ion emission from various Plasma-Focus (PF) facilities were performed in many laboratories all over the world, and their results were published in numerous papers which can be found as references in some review papers [1-5]. Such studies have also been carried out within different PF-type facilities in Poland [6-15]. It should be noted, however, that almost all PF devices mentioned above were operated at static initial gas conditions, and only a few of the PF-experiments were performed with an additional gas-puffing [16, 17]. However, the recent upgrading of the DPF-1000U facility operated at the IPPLM, Warsaw, and in particular the installation of a gas-puffing system, demanded new measurements of ion beams emitted at the changed experimental conditions to be performed. Hence, the main aim of the described study was to investigate the effects that the additional gas-puffing (through an axial pulsed gas-valve) had on the ion emission from the DPF-1000U facility. 


\section{Experimental setup}

The measurements described in this paper were performed with the use of the DPF-1000U device, which is equipped with two coaxial electrodes $460 \mathrm{~mm}$ long. The inner electrode is a copper tube $230 \mathrm{~mm}$ in diameter, while the outer electrode is composed of twelve symmetrically distributed stainless-steel pipes, forming a cylinder $400 \mathrm{~mm}$ in diameter. In the centre of the inner electrode a fast-acting gas valve was placed with a conical-shaped nozzle. That valve was used to inject additional gas (about $1 \mathrm{~cm}^{3}$, at the pressure of $0.5 \mathrm{bar}$ ) along the symmetry axis of the facility, in the region close to the front-plate of the inner electrode. During the measurements described below the gas valve was triggered $1.5 \mathrm{~ms}$ before the start of the main discharge. Plasma-focus discharges were initiated at the initial deuterium pressure of 1.6 or $2 \mathrm{hPa}$ by the application of a high-voltage pulse from the main condenser bank charged to $23 \mathrm{kV}$, which supplied the energy of about $334 \mathrm{~kJ}$. For the sake of comparison of ion emission characteristics the images of ions were recorded with ion pinhole cameras both for discharges with and without the use of gas-puffing. The mass- and energy-analyses were performed by means of a miniature Thomson-type spectrometer, mainly for discharges with the gas-puffing [15]. A diagnostic system at the DPF-1000U facility included also four silver neutron-activation counters which were used to estimate the total neutron yield in each discharge.

The ion pinhole camera or the spectrometer inlet were placed on the z-axis at a distance of $160 \mathrm{~cm}$ from the electrode outlets. Both diagnostic devices were equipped with nuclear track detectors of the PM-355 type. Magnification of the camera was chosen in such a way that the image of the outer electrodes could be observed, i.e., the distance between the pinhole inlet and the detector plane was either 4 or $7 \mathrm{~cm}$. The applied detectors could record ions of energies above $30 \mathrm{keV}$. The use of additional Al-foil filters of thickness equal to 0.75, 1.5 and $3 \mu \mathrm{m}$ allowed to shield the detectors and increase the detection threshold for deuterons - up to energies equal to 125,220 and $380 \mathrm{keV}$, respectively, according to the known transmission of the applied filters [18].

After the exposition during one or several shots the irradiated detectors were etched under standard conditions for 2 hours.

\section{Experimental results}

In order to analyze the recorded structure of the produced ion beams the detectors after their irradiation and etching were examined by means of an optical microscope equipped with a CCD camera and positioning table. The system made it possible to scan the surface of the detector and collect ion track images from the chosen area. The obtained images of ion tracks were analyzed using an image processing software [19] in order to determine the number and the nature of the recorded ion tracks.

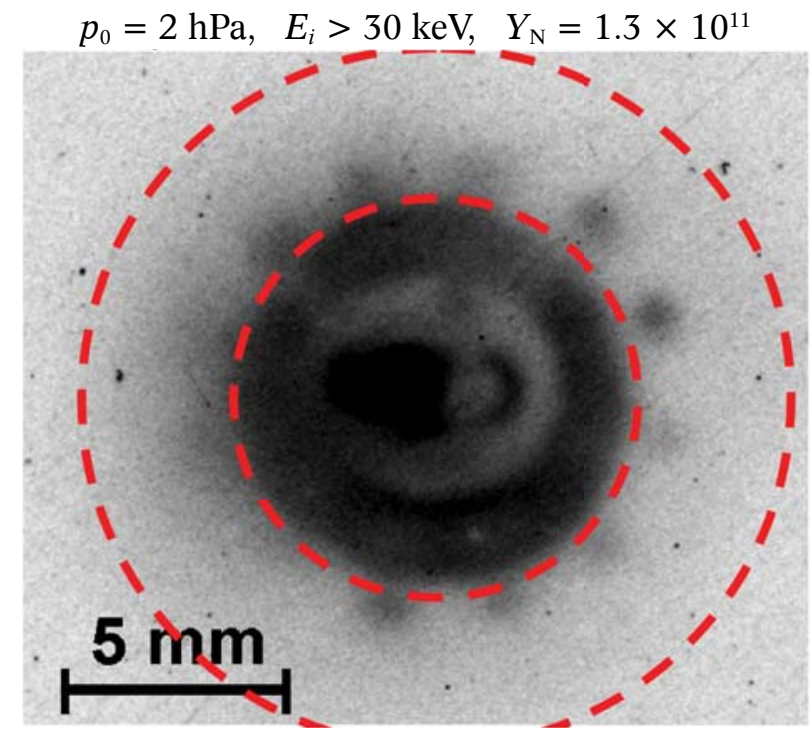

Fig. 1. The ion image obtained from the two successive DPF-1000U discharges which were performed without gas-puffing and which produced a relatively high neutron yield. Broken circles show projections of the electrodes on the detector plane. Ions with energies above $30 \mathrm{keV}$ were emitted mainly from the pinch column and the inter-electrode region.

An example of an ion image obtained from two successive DPF-1000U discharges without using the gas-puffing is shown in Fig. 1.

In this case the use was made of a pinhole camera with a smaller magnification. The detector was not shielded with any filter. The ion image consisted of a central bunch and annular streams corresponding to the inter-electrode gap.

To study the effects of the gas-puffing on the ion emission the use was made of a fast-acting axial valve situated behind an axial hole in the front plate of the inner electrode. The gas valve was triggered exactly $1.5 \mathrm{~ms}$ before the initiation of the discharge. In this case the ion measurements were performed at the initial deuterium pressure in the vacuum chamber equal to $1.6 \mathrm{hPa}$. An example of the ion images recorded without the use of any filter is shown in Fig. 2.

The upper image in Fig. 2, which was obtained from a discharge with a relatively high neutron yield, demonstrated an asymmetrical central ion bunch and a ring-shaped structure. Deuterons and protons with energies above $30 \mathrm{keV}$, were recorded in the form of numerous micro-beams, originated from the pinch column and the region between the electrodes, respectively. On the lower ion image, which was obtained from a discharge with a lower neutron yield, one can observe a ring-shaped structure only, without any central spot.

Further measurements involving irradiation of PM-355 detectors shielded with chosen filters made it possible to investigate the spatial structure of the ion emission and estimate the total numbers of ions having energies higher than the chosen energy threshold. An example of the ion density map, as obtained with such a shielded detector, is presented in Fig. 3 .

In this case the ions were recorded behind an $1.5 \mu \mathrm{m}$ thick Al-foil filter, so the energy threshold for detection of deuterons was $220 \mathrm{keV}$. The total 

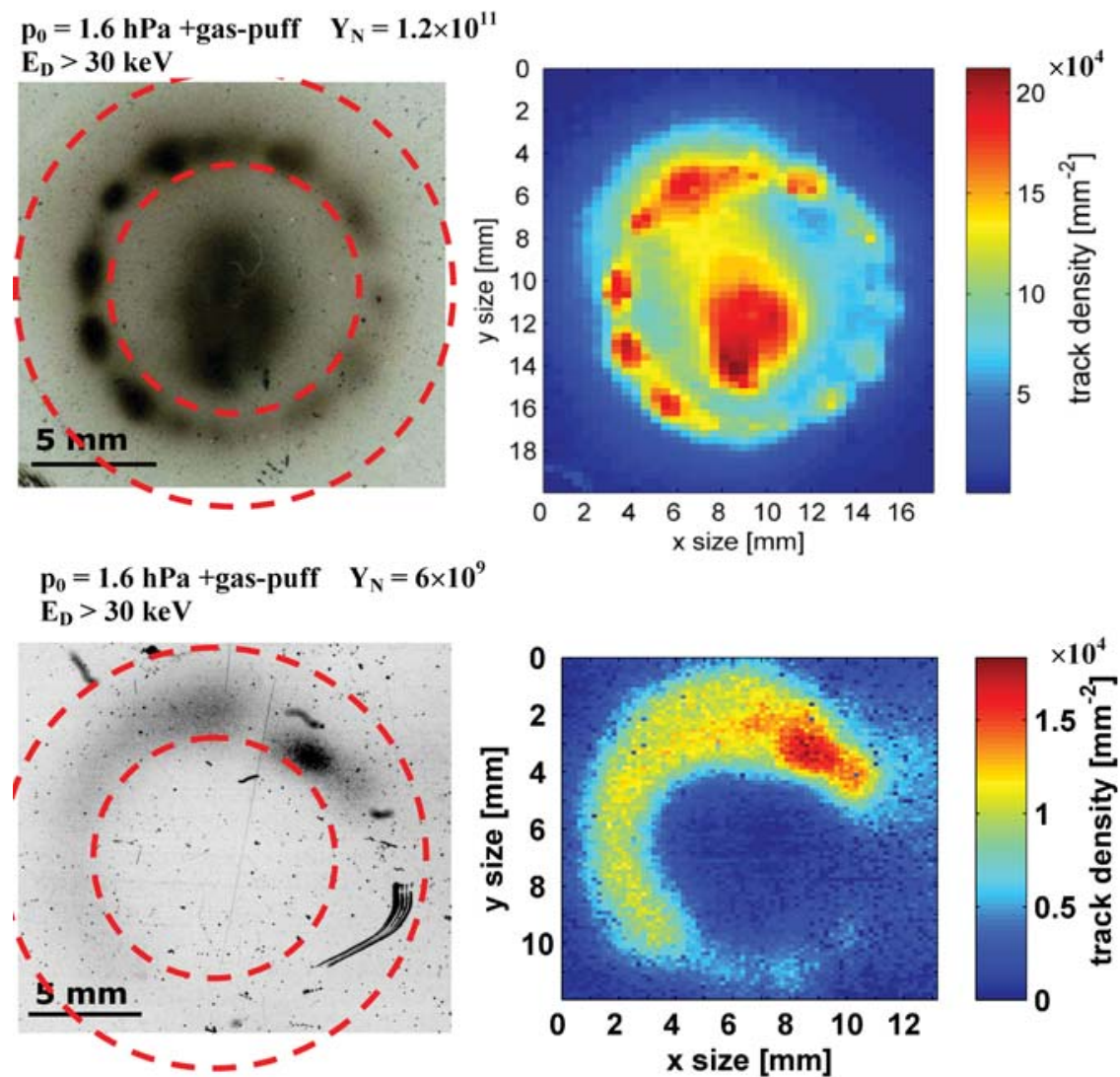

Fig. 2. Ion images obtained from two discharges with the use of the gas-puffing, recorded with detectors not covered by any filter (left column). The upper image was recorded at a high neutron yield, while the lower one - at a low neutron yield. The corresponding ion-density maps, as measured by means of microscope scanning, are shown in the right column.

neutron yield was relatively low (it amounted to $6.6 \times 10^{10}$ ) and the ion image could hardly be observed with the naked eye. The microscopic scanning has demonstrated nevertheless several micro-beams distributed around the centre of the detector. Another ion image recorded behind the $1.5 \mu \mathrm{m}$ Al-filter is shown in Fig. 4. In that case the total neutron

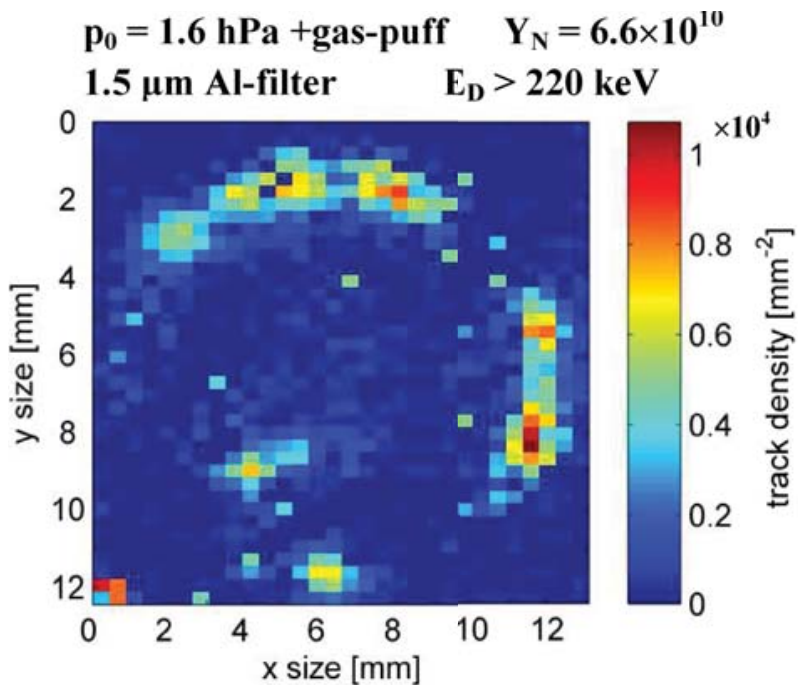

Fig. 3. The ion density map obtained from a single DPF-1000U discharge performed with the gas-puffing, which produced a lower neutron yield $\left(6.6 \times 10^{10}\right)$. The deuterons of energies above $220 \mathrm{keV}$ were recorded in a few small spots distributed on the external ring. yield was larger (about $1.4 \times 10^{11}$ ) and images of two intense deuteron beams (with a faint outer ring) could be observed.

An image created by deuterons with energies above $380 \mathrm{keV}$, which were recorded behind the $3 \mu \mathrm{m}$ Al-foil filter, is shown in Fig. 5. In that case a very intense and focused beam of high-energy ions was emitted from the pinch region, despite a relatively low total neutron yield (equal to about $6.7 \times 10^{10}$ ).

It should be noted that in certain areas of the irradiated detectors the density of the recorded tracks was too high to count tracks accurately; thus to estimate the total ion numbers it was necessary to use another method, the so-called binary processing. The results of such calculations, which were carried out for the detectors irradiated under different experimental conditions, are presented in Table 1.

Ion measurements performed with the Thomson spectrometer described above showed distinct deuteron- and proton-parabolas upon the irradiated and etched PM-355 detector. Such protons could originate from the hydrogen remnants which were previously absorbed by the electrodes and chamber walls. On the basis of the obtained images it was possible to calculate the energy spectra of the recorded ion beams (at the known energy calibration of the spectrometer).

The exemplary proton and deuteron energy spectra, as obtained from four successive discharges, are shown in Fig. 6. In that case the discharges were performed at the initial pressure equal to $1.6 \mathrm{hPa}$, 


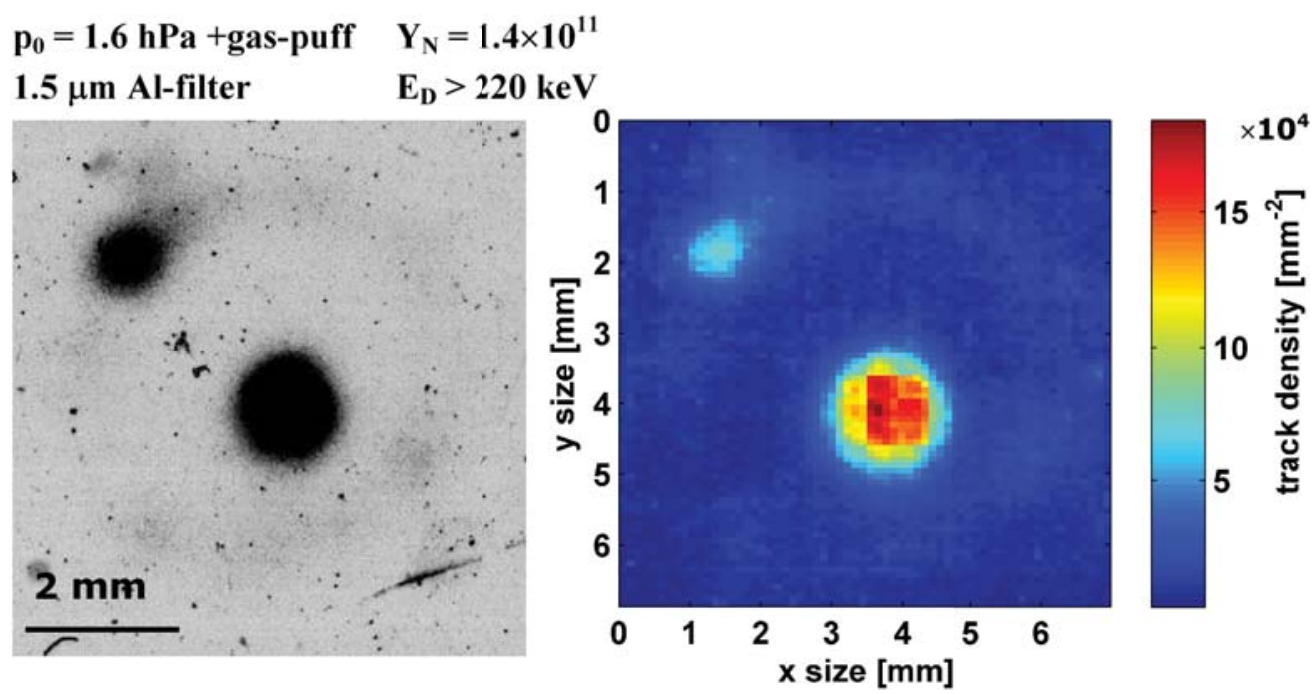

Fig. 4. The image and the density map of deuteron beams recorded for a single DPF-1000U discharge performed with the gas-puffing. The track detector was shielded with a 1.5- $\mu \mathrm{m}$ Al-foil filter which could be penetrated by deuterons with energies above $220 \mathrm{keV}$.

$$
\begin{array}{ll}
\mathrm{p}_{0}=1.6 \mathrm{hPa}+\text { gas-puff } & \mathrm{Y}_{\mathrm{N}}=6.7 \times 10^{10} \\
3 \mu \mathrm{m} \text { Al-filter } & \mathrm{E}_{\mathrm{D}}>380 \mathrm{keV}
\end{array}
$$
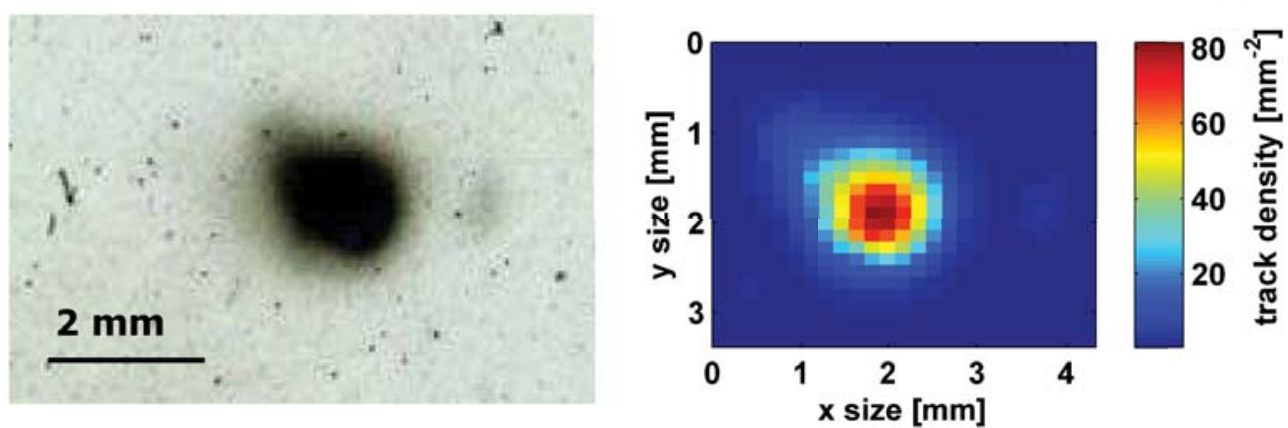

Fig. 5. The ion image and the corresponding density map recorded for another DPF-1000 discharge performed with the gas-puffing. The detector was shielded by a 3- $\mu \mathrm{m}$ Al-filter and the central beam was created by deuterons of energies above $380 \mathrm{keV}$.

with the use of gas-puffing, and the total neutron yield amounted to $2.9 \times 10^{11}$.

In order to estimate the energies of the deuterons leaving the plasma pinch column one should take into account the energy loss during their motion through a gas-filled region. Taking into consideration

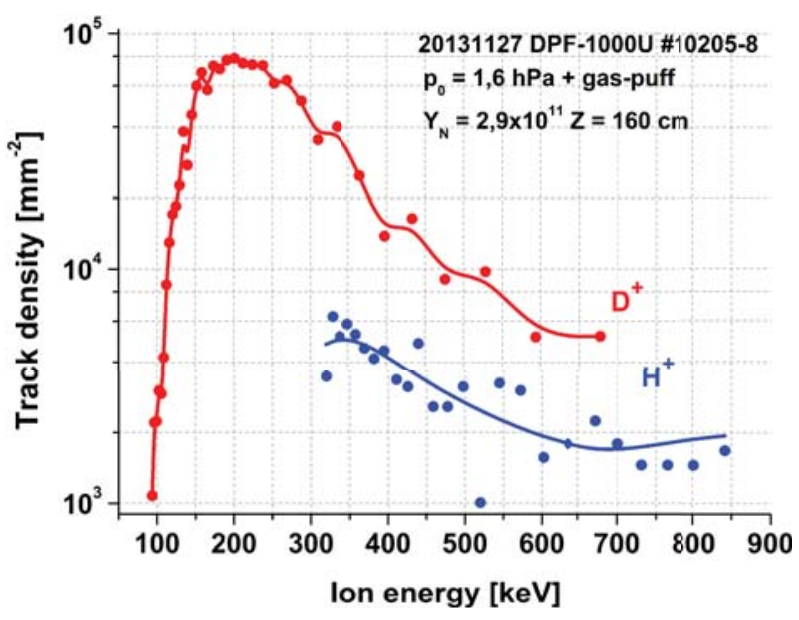

Fig. 6. Deuteron- and proton-energy spectra determined on the basis of four discharges performed at the initial deuterium pressure equal to $1.6 \mathrm{hPa}$ and the additional gas-puffing. the known experimental conditions (i.e. the distance from the plasma pinch and the deuterium filling pressures), it was possible to compute the ion energies as a function of the initial ion energy, as shown in Fig. 7. On the basis of this plot one can easily determine that deuterons of energies above 30,220 ,

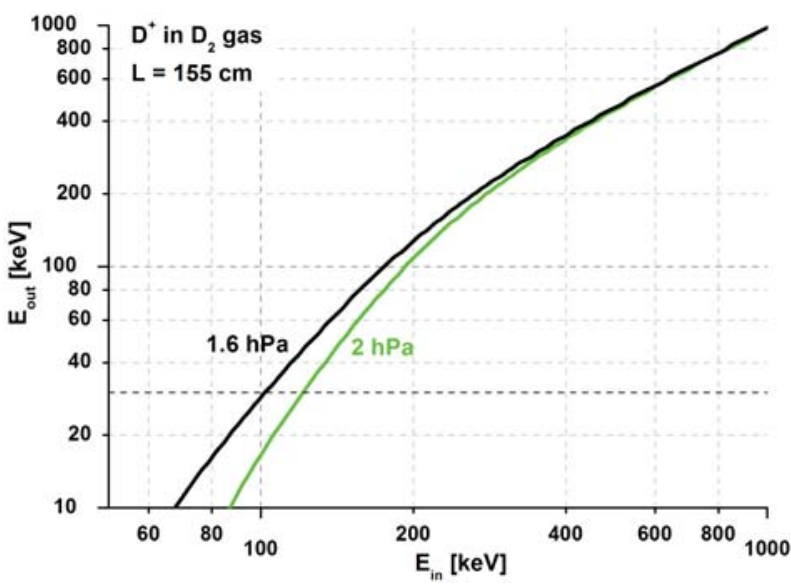

Fig. 7. Energies of deuterons recorded after passing a distance of $160 \mathrm{~cm}$ in the deuterium gas at the pressure of $1.6 \mathrm{hPa}$ or $2 \mathrm{hPa}$, as a function of their initial energy (when they leave the pinch region). 
Table 1. Parameters of the discharges and the number of ion tracks, as obtained for different detectors

\begin{tabular}{cccccc}
\hline Shot no. & $\begin{array}{c}\text { Deuterium } \\
\text { pressure }\end{array}$ & $\begin{array}{c}\text { Al filter } \\
{[\mu \mathrm{m}]}\end{array}$ & $\begin{array}{c}\text { Energy threshold } \\
{[\mathrm{keV}]}\end{array}$ & $\begin{array}{c}\text { Neutron } \\
\text { yield }\end{array}$ & $\begin{array}{c}\text { Number of ions } \\
( \pm 25 \%)\end{array}$ \\
\hline $10222-3$ & $2 \mathrm{hPa}$ & 0 & 30 & $1.3 \times 10^{11}$ & $2.0 \times 10^{6}$ \\
9971 & $1.6 \mathrm{hPa}+$ gas-puff & 0 & 30 & $1.2 \times 10^{11}$ & $10.0 \times 10^{6}$ \\
9933 & $1.6 \mathrm{hPa}+$ gas-puff & 0 & 30 & $6.0 \times 10^{9}$ & $0.7 \times 10^{6}$ \\
9936 & $1.6 \mathrm{hPa}+$ gas-puff & 1.5 & 220 & $1.4 \times 10^{11}$ & $0.4 \times 10^{6}$ \\
$10229-30$ & $1.6 \mathrm{hPa}+$ gas-puff & 1.5 & 220 & $6.6 \times 10^{10}$ & $0.15 \times 10^{6}$ \\
$9975-6$ & $1.6 \mathrm{hPa}+$ gas-puff & 3 & 380 & $6.7 \times 10^{10}$ & $0.7 \times 10^{6}$ \\
\hline
\end{tabular}

and $380 \mathrm{keV}$, which were recorded at a distance of $160 \mathrm{~cm}$ from the electrodes outlet, were emitted from the plasma pinch column with energies above 100, 160 , and $340 \mathrm{keV}$, respectively. It should be noted, however, that this is only a rough approximation of the initial ion energy values. In order to estimate the initial energies more accurately one should take into account the slowing-down of ions inside the dense plasma pinch and in the region of lower density plasma surrounding the pinch column.

The reported characteristics of ions in the detector plane appear to be of particular importance from the point of view of practical applications, e.g., the use of ion beams for modifications or erosion tests of different construction materials.

\section{Summary and conclusions}

Comparing the results obtained from the DPF-1000U discharges performed with the additional gas-puffing with the results of previous measurements $[13,14]$ one can conclude that the gas system does not have a major influence on the recorded ion pinhole images. The reported measurements of ions carried out with the ion pinhole cameras showed that the investigated ion beams have always a complex spatial structure. This structure consists of a central spot (bunch), generated by ions emitted along the pinch column, and an outer ring-shaped image, which is generated by ions emitted at different angles from the pinch column and the inter-electrode region.

It was shown that the outer ring-shaped image is formed by ions of lower energy than those in the central bunch. A relation between the total neutron yield and the obtained ion images suggests that the number of recorded ions increases with an increase in the neutron emission, but structures of the ion beams do not seem to be directly correlated with the total neutron yield.

The accelerated deuterons (primary ions), which are emitted during the DPF-1000U discharges with the gas-puffing, have energies up to $700 \mathrm{keV}$, while the fast protons (from the hydrogen remnants) have energies ranging from 300 to about $850 \mathrm{keV}$. It should be noted that these values of energy correspond to ions reaching the detector surface placed at a distance of $160 \mathrm{~cm}$ from the electrode ends. A comparison of the presented energy spectra with those recorded under stationary initial gas conditions, i.e. without the gas-puffing [13, 14], shows that despite the fact that protons can reach higher energies the deuteron energy spectra are comparable in both operational modes of the DPF-1000U facility.

It should be also noted that every plasma-focus discharge shows some unique features and ion images can differ in microscopic detail. Nevertheless, pictures of the ion bunches are reproducible on a macroscopic scale and can be compared. The obtained data are of importance not only for basic plasma physics, e.g., learning about the ion acceleration processes, but also for technological applications of the fast ion beams, e.g., in material engineering.

Acknowledgment. The described measurements of the fast ions within the DPF-1000U facility were partially motivated by preparations for laboratory tests of new ion probes, which were designed and manufactured within the framework of a grant from the Polish National Centre for Research and Development (NCBR) under a contract no. SP/J/2/1443234. This work was performed at IPPLM in Warsaw, Poland. This work was partly supported by the IAEA CRP RC-16954 and RC-16956 grants as well by the Polish Ministry of Science and Higher Education within the framework of the financial resources in the year 2014 allocated for the realization of the international co-financed projects.

\section{References}

1. Bertalot, L., Deutsch, R., Herold, H., Chuaqui, H., Gribkov, V., Herrera, J., Hirano, K., Krejci, A., Lee, S., Luo, F., Mezzetti, F., Sadowski, M., Schmidt, H., Ware, K., Wong, C. S., \& Zoita, V. (1981). Experiments on plasma focus dynamics, neutron production and ion emission. In Plasma Physics and Controlled Nuclear Fusion Research 1980. Proceedings of an International Conference, Brussels, 1-10 July 1980 (Vol. 2, pp. 177-185). Vienna: IAEA.

2. Herold, H., Jerzykiewicz, A., Sadowski, M., \& Schmidt, H. (1989). Comparative analysis of large plasma focus experiments performed at IPF-Stuttgart and at IPJ-Świerk. Nucl. Fusion, 29, 1255-1269. DOI: 10.1088/0029-5515/29/8/002.

3. Bernard, A., Bruzzone, H., \& Choi, P. (1998). Scientific status of plasma focus research. J. Moscow Phys. Soc., 8, 93-170.

4. Sadowski, M., Szydlowski, A., Scholz, M., Kelly, H., Marquez, A., \& Lepone, A. (1999). Application of solid-state nuclear track detectors for studies of fast ion beams within PF-1000 and Rother plasma-focus facilities. Radiat. Meas., 31(1), 185-190. DOI: 10.1016/S1350-4487(99)00083-9. 
5. Gribkov, V. A., Banaszak, A., Bienkowska, B., Dubrovsky, A. V., Ivanova-Stanik, I., Jakubowski, L., Karpinski, L., Miklaszewski, R. A., Paduch, M., Sadowski, M. J., Scholz, M., Szydlowski, A., \& Tomaszewski, K. (2007). Plasma dynamics in the PF-1000 device under full-scale energy storage: II. Fast electron and ion characteristics versus neutron emission parameters and gun optimization perspectives. J. Phys. D-Appl. Phys., 40(12), 3592-3607. DOI: 10.1088/0022-3727/40/12/008.

6. Sadowski, M., Żebrowski, J., Rydygier, E., \& Kuciński, J. (1988). Ion emission from plasma-focus facilities. Plasma Phys. Contr. Fusion, 30(6), 763-769. DOI: 10.1088/0741-3335/30/6/008.

7. Sadowski, M. (2000). Studies and applications of dense magnetized plasmas. J. Tech. Phys., Special Issue, 41(1), 123-145.

8. Jakubowski, L., Sadowski, M., \& Żebrowski, J. (2001). Measurements of charged particle beams from plasma-focus discharges. Nucl. Fusion, 41(6), 755-759. DOI: 10.1088/0029-5515/41/6/311.

9. Szydłowski, A., Scholz, M., Karpiński, L., Sadowski, M., Tomaszewski, K., \& Paduch, M. (2001). Neutron and fast ion emission from PF-1000 facility equipped with new large electrodes. Nukleonika, 46(Suppl. 1), S61-S64.

10. Sadowski, M., \& Zebrowski, J. (2002). Studies of pulsed ion- and electron-beams emitted from plasma discharges. J. High Temp. Mat. Proc., 6, 1-14. DOI: 10.1615/HighTempMatProc.v6.i1.10.

11. Sadowski, M. J., \& Scholz, M. (2003). Recent results of Large-Scale Plasma Focus experiment. In Proceedings 30th of EPS Conference on Contribution Fusion and Plasma Physics, 7-11 July 2003 (27A, P-1.207). St. Petersburg, Russia: ECA.

12. Sadowski, M. J., \& Scholz, M. (2008). The main issues of research on dense magnetized plasmas in $\mathrm{PF}$ discharges. Plasma Sources Sci. Technol., 17, 024001. DOI: 10.1088/0963-0252/17/2/024001.

13. Skladnik-Sadowska, E., Czaus, K., Kwiatkowski, R., Malinowski, K., Sadowski, M. J., Żebrowski, J., Karpinski, L., Paduch, M., \& Scholz, M. (2010) Recent studies of the ion emission from high-current PF-1000 experiments. Problems of Atomic Science and Technology, Seria: Plasma Phys., 6(16), 199-201.

14. Kwiatkowski, R., Skladnik-Sadowska, E., Malinowski, K., Sadowski, M. J., Czus, K., Zebrowski, J., Karpinski, L., Paduch, M., Scholz, M., Garkusha, I. E., \& Kubes, P. (2011). Measurements of electron and ion beams emitted from the PF-1000 device in the upstream and downstream direction. Nukleonika, 56(2), 119-123.

15. Czaus, K., Skladnik-Sadowska, E., Malinowski, K., Sadowski, M. J., Czaus, K., Zebrowski, J., Karpinski, L., Paduch, M., Scholz, M., Garkusha, I. E., Kubes, P. (2013). Progress in mass- and energy-analysis of ion beams emitted from RPI- and PF-type discharges. Problems of Atomic Science and Technology, Seria: Plasma Phys., 19, 261-263.

16. Sadowski, M. J., Baranowski, J., Jakubowski, L. Skladnik-Sadowska, E., \& Szydlowski, A. (1990). Investigation of gas puffed discharges of the Plasma Focus type. In Proceedings of 17th IEEE International Conference on Plasma Science (4P2-16). Oakland. DOI: 10.1109/PLASMA.1990.110794.

17. Schmidt, H., Sadowski, M. J., Jakubowski, L., Skladnik-Sadowska, E., \& Stanislawski, J. (1994). Gas-puff target experiment with the Poseidon plasma focus facility. Plasma Phys. Contr. Fusion, 36, 13. DOI: 10.1088/0741-3335/36/1/002.

18. Ziegler, J. F., Biersack, J. P., \& Ziegler, M. D. (2012). SRIM Textbook. James Ziegler.

19. Ferreira, T., \& Rasband, W. (2012, October). Image J User Guide. Retrieved March 10, 2013. http://imagej. nih.gov/ij/docs/guide/index.html. 